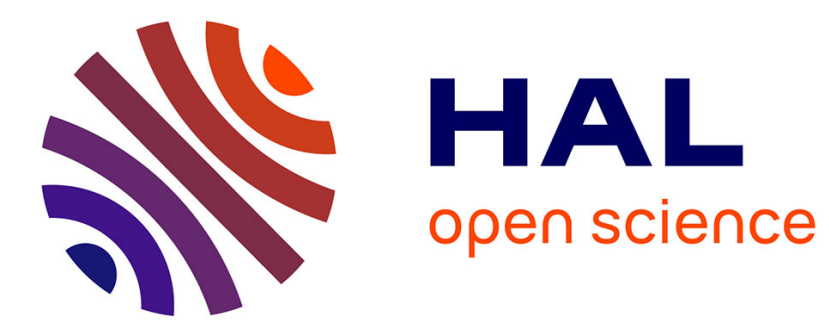

\title{
Self-eclipsing: alignment-free vortex coronagraphy
}

Artur Aleksanyan, Etienne Brasselet

\section{To cite this version:}

Artur Aleksanyan, Etienne Brasselet. Self-eclipsing: alignment-free vortex coronagraphy. Optics Letters, 2017, 42 (7), pp.1237-1240. 10.1364/OL.42.001237 . hal-01494646

\section{HAL Id: hal-01494646 \\ https://hal.science/hal-01494646}

Submitted on 23 Mar 2017

HAL is a multi-disciplinary open access archive for the deposit and dissemination of scientific research documents, whether they are published or not. The documents may come from teaching and research institutions in France or abroad, or from public or private research centers.
L'archive ouverte pluridisciplinaire HAL, est destinée au dépôt et à la diffusion de documents scientifiques de niveau recherche, publiés ou non, émanant des établissements d'enseignement et de recherche français ou étrangers, des laboratoires publics ou privés.

\section{두(1)}

Distributed under a Creative Commons Attribution - ShareAlikel 4.0 International 


\title{
Self-eclipsing: alignment-free vortex coronagraphy
}

\author{
Artur Aleksanyan ${ }^{1,2}$ and Etienne Brasselett ${ }^{1,2, *}$ \\ ${ }^{1}$ Université de Bordeaux, LOMA, UMR 5798, F-33400 Talence, France \\ ${ }^{2}$ CNRS, LOMA, UMR 5798, F-33400 Talence, France \\ *Corresponding author: etienne.brasselet@u bordeaux.fr
}

\begin{abstract}
We report on a self-induced strategy to achieve highcontrast optical imaging, without the need for any manmade optical masks, which relies on the self-induced spin-to-orbital angular momentum conversion phenomenon. This is experimentally demonstrated by realizing a laboratory demonstration of self-eclipsing of a light source following the generation of a self-adapted vectorial optical vortex transmission mask. The proposed concept, namely the realization of an alignment-free optical vortex coronagraph, may inspire the development of future generations of smart astronomical imaging instruments.
\end{abstract}

OCIS codes: (350.5340) Photothermal effects; (050.4865) Optical vortices.

In searching and understanding extrasolar planets, starting from the pioneering observation by Mayor and Queloz [1], the de velopment of high contrast optical imaging techniques, such as coronagraphs, is a primary topic of modern observational astro physics [2]. In addition to the continued development of adap tive optics and detectors with better performance, there is a constant need to realize optical phase and/or amplitude masks with ever increasing complexity. This is especially true in the context of future large telescopes and space missions that re quire the development of instruments with demanding speci fications and whose technological solutions remain so far under study.

Among possible options that have been developed since the era of stellar coronagraph initiated by Lyot in the 1930s [3], optical vortex coronagraphs have emerged in the 2000s $[4,5]$, whose vectorial version has been found to be promising enough to equip several large telescopes worldwide [6]. A vec torial vortex coronagraph basically relies on a space variant anisotropic phase mask characterized by a birefringent phase retardation $\Delta=\pi$ and a spatial distribution of the optical axis orientation angle of the form $\psi=m \phi$, with $\phi$ being the polar angle in the plane of the mask with a $m \geq 1$ integer, which leads to the generation of optical vortices with topological charge $\pm 2 \mathrm{~m}$. After a few years of on sky harvest using vectorial vortex coronagraphs of fundamental order $m=1$ [6], astrono mers now work toward the implementation of high charge vec torial vortex coronagraphs, namely $m \geq 2$ [7]. In practice, the case $m=2$ appears as a good compromise between an enhanced pointing stability and a small inner working angle. Such high order masks have been already realized using liquid crystal poly mer technology and tested on high contrast imaging testbeds [8], and since then, the technology has been improved $[9,10]$. Still, large spectral bandwidth coronagraphs remain an important issue deserving continuous development, in particular using various vectorial approaches, such as the polymer liquid crystal technol ogy $[11,12]$ and the subwavelength gratings technology [13]. Exploring new avenues to achieve a smart stellar light rejection is the context of the present work, which considers the case of vectorial vortex coronagraph with $m=1$.

Recently, a nature assisted approach to realize high resolution liquid crystal vectorial vortex coronagraph of order $m=1$ made of self engineered topological defects has been proposed [14]. However, although the latter attempt is en dowed with good coronagraphic performances compared to that of the state of the art artificial strategies [6], they remain endowed with major drawbacks toward practical implementa tion. First, the created phase masks randomly appear, and uncontrollable residual drifts may occur due to the liquid crystalline nature of the used materials, which prevents imple mentation in large telescopes. Second, the spatial extent of the appropriately structured birefringent material is usually lim ited to a few tens of microns. Solving these issues while preserving self engineered features would generate the potential to foster novel generation of smart astronomical imaging instruments.

In this context, here we propose and experimentally report on the laboratory demonstration of the self eclipsing of a bright source that leaves a faint nearby companion almost unaffected. Namely, the bright source creates its own vectorial optical vortex transmission mask inside an isotropic absorptive medium via a photoelastic phenomenon driven by thermal radial gradients. Remarkably, since the latter effect is reversible, our alignment free vortex mask has real time self adaptation capabilities; hence it is naturally resilient to low frequency pointing noise. 
For the purpose of demonstration, we use a simple slab of absorptive glass (from Thorlabs) as the photoactive singular optical element, with thickness $L=1.89 \mathrm{~mm}$ and optical den sity 1 , while light sources are prepared from a continuous wave diode pumped solid state laser with wavelength $532 \mathrm{~nm}$. According to Ref. [15], a light beam passing through such a slab generates a vectorial vortex mask with $m=1$, whose birefringent phase retardation depends on the distance $r$ to the beam axis, $\Delta(r)$. In the limit of small absorption (that is, a $z$ independent heat deposition in the bulk of the medium) and under the cylindrical beam approximation, an analytical profile for $\Delta(r)$ can be obtained assuming a Gaussian heat source, namely [16]

$$
\Delta(r)=\Delta_{\infty} F\left(r / w_{0}\right),
$$

with

$$
F(x)=1+\frac{\exp \left(2 x^{2}\right) \quad 1}{2 x^{2}},
$$

where $\Delta_{\infty}$ is the asymptotic value at large $r$, and $w_{0}$ is the waist radius of the incident laser beam with an intensity profile $I(r)=I(0) \exp \left(2 r^{2} / w_{0}^{2}\right)$. Since the incident beam is prepared by focusing the output field of an uniformly illuminated iris of radius $R_{1}=1 \mathrm{~mm}$ by a lens with focal length $f_{1}=500 \mathrm{~mm}$, see Fig. 1(a), the numerical aperture is $\mathrm{NA}=0.002$. Moreover, we note that the cylindrical beam approximation implies $L \ll z_{0}$ where $z_{0}=\pi w_{0}^{2} / \lambda$ is the Rayleigh distance of the Gaussian beam. In our case, the beam profile in the focal plane of the lens $f_{1}$ is not a Gaussian spot but an Airy spot with an intensity profile $I(r)=4 I(0)\left|J_{1}\left(k R_{1} r / f_{1}\right) /\left(k R_{1} r / f_{1}\right)\right|^{2}$, where $k=2 \pi / \lambda$

(a)
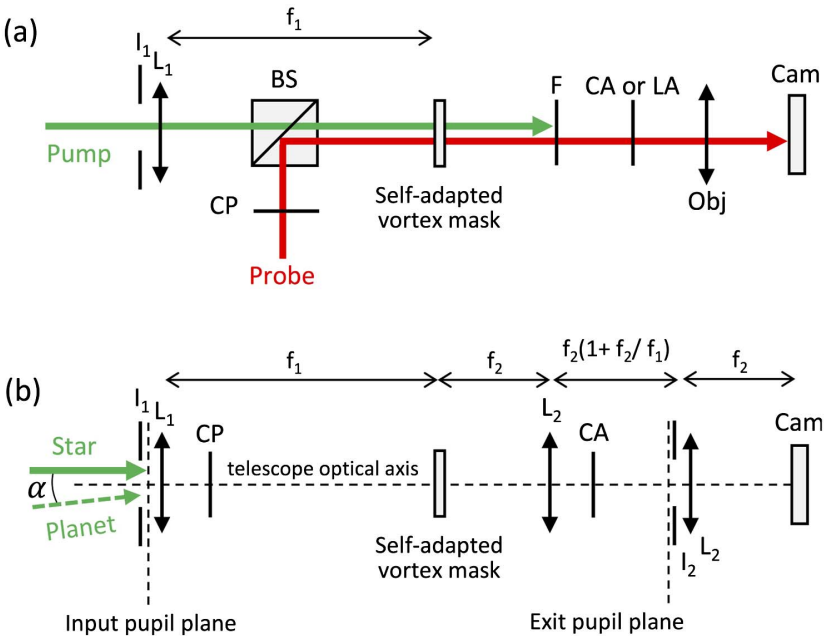

Fig. 1. (a) Setup for creating the self adapted vectorial vortex mask of order $m=1$. $I_{1}$, iris with radius $R_{1}=1 \mathrm{~mm} ; L_{1}$, lens with focal length $f_{1}=500 \mathrm{~mm}$; $\mathrm{BS}$, beam splitter; $\mathrm{CP}$, circular polarizer; $F$, notch filter at $532 \mathrm{~nm}$ wavelength that blocks the pump beam while fully transmitting the probe beam; CA, circular analyzer for the mea surement of $\Delta(r)$; LA, linear analyzer for the measurement of $\psi(\phi)$; Obj, microscope objective $(\times 4, \mathrm{NA}=0.1)$; and Cam, camera. (b) Setup for the demonstration of alignment free optical vortex co ronagraphy. The two beams labeled "star" and "planet" have an angular separation $\alpha$, with the starlight propagation direction aligned with the telescope axis. $I_{2}$, iris with radius $R_{2}^{\prime}=0.75\left(f_{2} / f_{1}\right) R_{1}$ and $L_{2}$, lens with focal length $f_{2}=300 \mathrm{~mm}$. and $J_{1}$ is the first order Bessel function of the first kind. In fact, an Airy pattern can be locally described to a good approximation by an effective Gaussian profile with a waist radius $w_{\text {eff }} \simeq 0.7 r_{0}$, where $r_{0}=0.61 \lambda / \mathrm{NA} \simeq 160 \mu \mathrm{m}$ is the first zero of the intensity profile, which is obtained by perform ing a fit over the range $0 \leq r / r_{0} \leq 1.25$. Thus, one obtains $z_{0} \simeq 7 \mathrm{~cm}$, which validates the approximation $L \ll z_{0}$.

The creation of a self adapted vortex mask with $m=1$ is experimentally assessed by probing the glass slab with a spatially extended collimated laser beam at $633 \mathrm{~nm}$ wavelength, as shown in Fig. 1(a). The incident polarization state is set as cir cular and the intensity profile of the orthogonally polarized component of the output field is recorded by a camera via imaging with a microscope objective, see Fig. 2(a). The ob tained intensity profile between crossed circular polarizers is ex pressed as $I(r) / I_{\infty}=\sin ^{2}[\Delta(r) / 2] / \sin ^{2}\left[\Delta_{\infty} / 2\right]$, where $I_{\infty}$ is the asymptotic value at large $r$. In practice, the power of the generated vortex beam is only a small fraction of the output light field; indeed, we measured the latter fraction to be on the order of $0.1 \%$. Therefore, the above expression of the re duced intensity radial profile can be safely approximated by the universal profile $I(r) / I_{\infty}=F^{2}\left(r / w_{0}\right)$. A satisfying agreement is obtained between the experimental data, see markers in Fig. 2(b), and the expression given by Eq. (1), see solid curve in Fig. 2(b), a good match being found for $w_{0}=75 \mu \mathrm{m}$ that should be compared with the effective Gaussian waist $w_{\text {eff }} \simeq 110 \mu \mathrm{m}$. Such a discrepancy could be attributed to the Gaussian heat source assumption, but it is more likely because our experimental conditions (10\% power transmis sion) go beyond the approximation of a $z$ independent bulk heat deposition [16]. Alternatively, the expected spatial distri bution of the optical axis orientation angle $\psi=\phi$ is also (a)

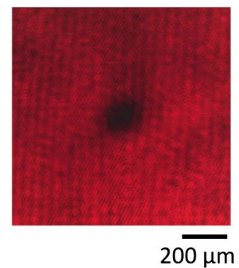

(c)

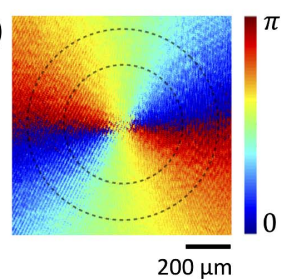

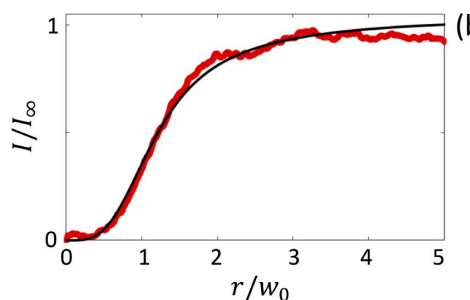

(b)

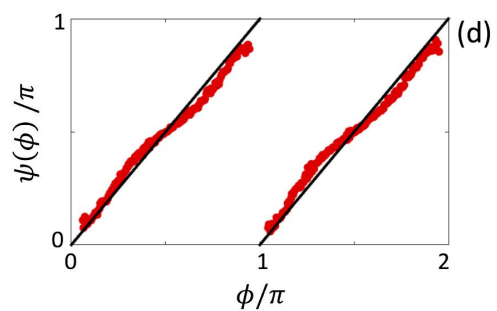

Fig. 2. (a) Typical experimental image of the self adapted vortex mask observed between crossed circular polarizers for an incident pump beam power $P \simeq 190 \mathrm{~mW}$. (b) Markers: measurement of the reduced radial intensity profile of the self generated vortex beam $I / I_{\infty}$ from the data shown in panel (a), in spatial units of $w_{0}$. Solid curve: theoretical dependence given by the function $F^{2}$, see Eq. (2). (c) Typical experimental map of the spatial distribution of the light induced optical axis orientation angle $\psi$. (d) Markers: measured $\psi(\phi)$ from the radial average between the two dashed circles shown in panel (c). Solid curve: theoretical dependence expected from a vectorial vortex mask with charge $m=1$. 

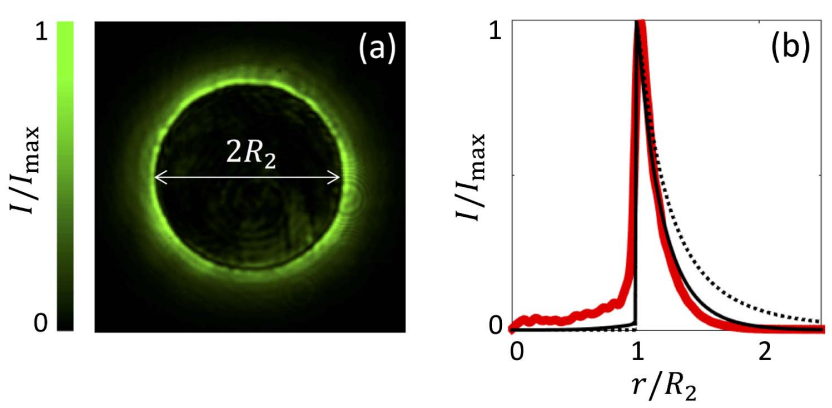

Fig. 3. (a) Self eclipsing of the starlight observed by placing the camera at the exit pupil plane for an incident power $P \simeq 50 \mathrm{~mW}$. (b) Thick solid red curve: azimuth average radial intensity profile of the data shown in panel (a). Thin solid black curve: calculated in tensity profile based on the self adapted vortex mask model used here. Thin dotted black curve: calculated intensity profile for a vectorial vortex mask with ideally uniform birefringent phase retardation.

retrieved experimentally from the reduced Stokes parameters $s_{1}$ and $s_{2}$ [17] following $\psi=(1 / 2) \arctan \left(s_{2} / s_{1}\right) \quad \sigma \pi / 4$, where $\sigma= \pm 1$ refers to the helicity of the incident circularly polarized probe beam, see Figs. 2(c) and 2(d). The above measurements are made under pump incident power $P=192 \mathrm{~mW}$, which is large enough to ensure a satisfying signal to noise ratio after polarization filtering and gives $\Delta_{\infty} \sim 0.06$ for the pump wave length in the present case. We note that these results are inde pendent of the polarization state of the pump light, as expected form the thermal origin of the photoelastic effect in iso tropic media.

Next, we explore the coronagraphic capabilities of such self adapted vortex masks. This is conducted using the exper imental arrangement sketched in Fig. 1(b), which refers to the typical setup for the laboratory demonstration of a corona graph, where the starlight is mimicked by an on axis incident quasi plane wave clipped by the input pupil of the telescope defined by the iris $I_{1}$. Here, the starlight generates its own vec torial vortex mask, which leads to a self eclipsing phenomenon in the exit pupil plane located at a distance $f_{2}\left(1+f_{2} / f_{1}\right)$ from the output of the telescope made of two lenses of focal lengths $f_{1}$ and $f_{2}$, see Fig. 1(b). We note that since the self generated vector vortex mask is far from ideally efficient (see $0.1 \%$ vortex generation purity mentioned above), the practical validation of the proposed concept implies the use of crossed circular polarizers observations, see Fig. 1(b).

The recorded image of the self eclipse is shown in Fig. 3(a). The corresponding radial intensity profile is com pared to the prediction of the model given under the approxi mation of paraxial wave propagation. Namely, $I_{\text {exit pupil }}=$ $\mid \mathcal{F}^{-1}\left[\left.\tau(r, \phi) \mathcal{F}\left[E_{\text {input pupil }}\right]\right|^{2}\right.$, where $\mathcal{F}\left(\mathcal{F}^{-1}\right)$ refers to the (inverse) Fourier transform and $E_{\text {input pupil }} \propto \operatorname{circ}\left(r / R_{1}\right)$ refers to the uniformly illuminated circular input pupil plane, with $\operatorname{circ}(r / \rho)=0$ for $r>\rho$ and 1 for $r<\rho$. In addition, the complex amplitude transmittance of the self adapted vortex mask is, up to a constant factor [14],

$$
\tau(r, \phi)=\sin [\Delta(r) / 2] \exp [2 i \phi+i \Delta(r) / 2] .
$$

As shown in Fig. 3(b), we obtain a satisfying match between the measured (thick red solid curve) and calculated (thin black (a)
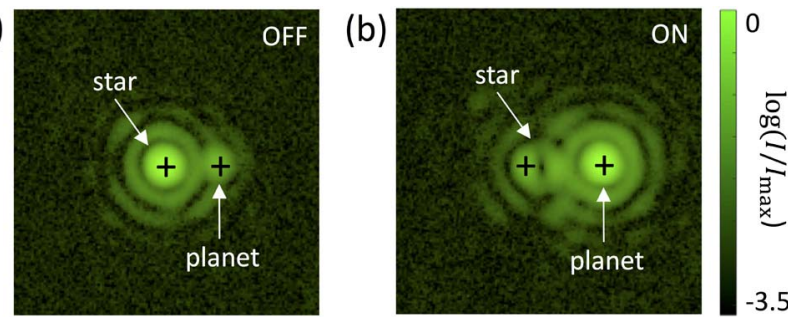

Fig. 4. Laboratory demonstration of alignment free optical vortex coronagraphy of a star/planet binary source with $2 \alpha_{\text {diff }}$ angular sepa ration, using a 12 bit camera. Panels (a) and (b) refer to experimental data when the coronagraph is OFF (a) and ON (b). Here the incident power of the star source is $\simeq 50 \mathrm{~mW}$, which is 10 times larger than that of the secondary source. Scale bar applies for all four panels with $I_{\max }$ being the maximal intensity value of each panel.

solid curve) data, where we used the experimentally determined value, $w_{0}=75 \mu \mathrm{m}$, for the simulations. For comparison, we have added on the latter figure the expected radial intensity profile associated with an ideally uniform vortex mask de scribed by a complex transmittance $\tau_{\text {ideal }}(\phi)=\exp (2 i \phi)$ giving $I / I_{\max }=0$ if $r<R_{2}$ and $I / I_{\max }=\left(R_{2} / r\right)^{2}$ if $r>R_{2}$ [5] (thin black dotted curve) with $R_{2}=\left(f_{2} / f_{1}\right) R_{1}$. Such a com parison enables appreciation that the non uniform birefringent phase retardation profile inherent to the photoelastic effect must be taken into account for a proper quantitative descrip tion of the self eclipsing phenomenon.

By placing a so called Lyot stop [namely, the iris $I_{2}$, see Fig. 1(b)] in the exit pupil plane, one can selectively reject the on axis starlight while the less intense off axis light from a "planet" is expected to be unaltered by the self adapted vortex mask created by the star. Practically, this is made by using a Lyot stop with radius $R_{2}^{\prime}=0.75 R_{2}$, an incident star beam power 10 times larger than the incident planet beam power and an angular separation of $\simeq 2 \alpha_{\text {diff }}$ between the star and planet light sources, where $\alpha_{\text {diff }}=0.61 \lambda / R_{1}$ is the diffraction limit associated with the circular entrance pupil aperture of radius $R_{1}$. This gives a power rejection rate, defined as the ratio between the power out side and inside the Lyot stop, of $\simeq 40$. Next, the coronagraphic capabilities are tested by reimaging the star and planet on the camera by the lens $L_{2}$, see Fig. 1(b). The experimental results are displayed in Figs. 4(a) and 4(b). The former figure corre sponds to the situation just after the illumination is switched on, namely the vortex mask is not activated, which refers to the OFF state of the coronagraph. In contrast, the latter figure corresponds to the steady state situation when the self adapted vortex mask is formed and stationary, which thus refers to the ON state of the coronagraph. As expected, self eclipsing enables the achievement of high contrast imaging, which is quantified by comparing the values of the ratio $\zeta=I_{\text {star }}^{\max } / I_{\text {planet }}^{\max }$ when the self coronagraph is OFF and ON, where $I_{\text {star }}^{\max }$ and $I_{\text {planet }}^{\max }$ refer to the local intensity maxima associated with the star and planet, see black crosses shown in Figs. 4(a) and 4(b). We found $\zeta_{\mathrm{OFF}} \simeq 10$ and $\zeta_{\mathrm{ON}} \simeq 0.1$, which corresponds to a peak to peak contrast improvement by a factor $\simeq 100$ that is a decent value when compared with reported values using standard corona graphs $[6,11,18]$.

In the present situation, the characteristic time to reach the steady state regime is of the order of a few seconds. This is 
Excitation dynamics

(a)
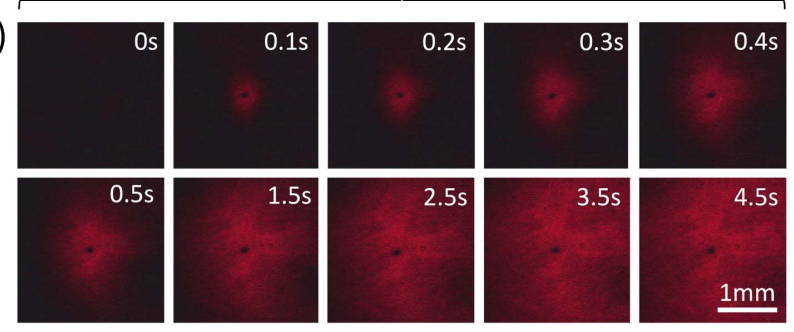

Relaxation dynamics

(b)
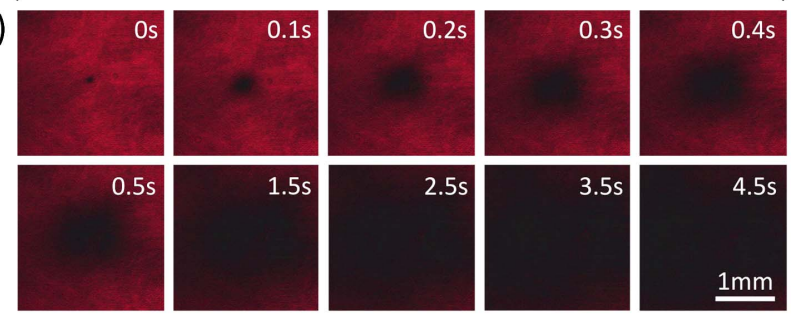

Fig. 5. (a) Excitation and (b) relaxation dynamics of the self adapted vectorial vortex mask monitored by imaging the sample between crossed circular polarizers following the setup shown in Fig. 1(a) under pump incident power $P \simeq 50 \mathrm{~mW}$.

illustrated in Fig. 5(a) where the dynamics of the self induced vortex mask observed between crossed circular polarizers is shown at different times $t$, the laser being switched on at $t=0$, for on axis illumination with incident power $P \simeq$ $50 \mathrm{~mW}$ according to the setup shown in Fig. 1(a). The relax ation takes place over similar time scale, as shown in Fig. 5(b). Note that Fig. 5 emphasizes large scale material structuring (here a couple of millimeters), which is another advantage of the proposed mechanism associated with the nonlocal nature of the photoelastic effect. Moreover, since the photoelastic ef fect is a reversible phenomenon, the proposed alignment free approach to vectorial vortex coronagraphy is thus endowed with real time self adaptation capabilities. In other words, such a self coronagraph is naturally resilient to low frequency time dependent pointing, which offers a novel strategy to long term observations without source tracking. One may argue that the low level photon flux associated with on sky astronomical ap plications cannot trigger a photoelastic effect, enabling the generation of a self adapted vortex mask. However, one may realistically consider the implementation of a source tracking feedback mechanism ensuring local heating of a slab of an iso tropic material transparent to the observation wavelength. For instance, by laser heating at a wavelength associated with an absorption band or by electrical heating using localized trans parent electrode coated on the slab, which would have the ad vantage to not compromise the throughput of the instrument. Another aspect that is worth mentioning is the chromaticity; indeed, stellar coronagraphs are ideally required to operate over large bandwidth. In the present case, the reported effect corre sponds to a space variant stress induced birefringent phase retardation that scales as $\propto 1 / \lambda$ (neglecting the dispersion of the induced optical anisotropy), which implies wavelength dependent performances.
In conclusion, we have explored an original high contrast imaging strategy where the light source that should be rejected creates its own, self adapted, vortex mask. In other words, we unveiled a self eclipsing phenomenon. The effect relies on a self induced spin to orbital angular momentum conversion phenomenon driven by the photoelastic effect in absorptive iso tropic media. Although we are aware that there is a practical gap toward a direct implementation of such an alignment free coronagraph for astronomical observations without the assis tance of a feedback phenomenon enabling the local heating of the sample, we believe that the proposed concept may stimu late the development of smart alternatives to mainstream high contrast imaging instruments.

Funding. Agence Nationale de la Recherche (ANR) (ANR 10 IDEX 03 02, ANR 15 CE30 0018).

Acknowledgment. This study has been carried out with financial support from the French State, managed by the French National Research Agency (ANR) in the frame of the Investments for the future Programme IdEx Bordeaux LAPHIA and HYPERPHORB project.

\section{REFERENCES}

1. M. Mayor and D. Queloz, Nature 378, 355 (1995).

2. F. Pepe, D. Ehrenreich, and M. R. Meyer, Nature 513, 358 (2014).

3. B. Lyot, Mon. Not. R. Astron. Soc. 99, 580 (1939).

4. D. Mawet, P. Riaud, O. Absil, and J. Surdej, Astrophys. J. 633, 1191 (2005).

5. G. Foo, D. M. Palacios, and G. A. Swartzlander, Opt. Lett. 30, 3308 (2005).

6. O. Absil, D. Mawet, M. Karlsson, B. Carlomagno, V. Christiaens, D. Defrére, C. Delacroix, B. Femenìa Castella, P. Forsberg, J. Girard, C. A. Gómez González, S. Habraken, P. M. Hinz, E. Huby, A. Jolivet, K. Matthews, J. Milli, G. Orban de Xivry, E. Pantin, P. Piron, M. Reggiani, G. J. Ruane, G. Serabyn, J. Surdej, K. R. W. Tristram, E. Vargas Catalán, O. Wertz, and P. Wizinowich, Proc. SPIE 9908, 99080Q (2016).

7. C. Delacroix, C. Delacroix, O. Absil, B. Carlomagno, P. Piron, P. Forsberg, M. Karlsson, D. Mawet, S. Habraken, and J. Surdej, Proc. SPIE 9147, 91478Y (2014).

8. D. Mawet, L. Pueyo, D. Moody, J. Krist, and E. Serabyn, Proc. SPIE 7739, 773914 (2010).

9. S. R. Nersisyan, N. V. Tabiryan, D. Mawet, and E. Serabyn, Opt. Express 21, 8205 (2013).

10. J. Kim, Y. Li, M. N. Miskiewicz, C. Oh, M. W. Kudenov, and M. J. Escuti, Optica 2, 958 (2015).

11. D. Mawet, E. Serabyn, K. Liewer, R. Burruss, J. Hickey, and D. Shemo, Astrophys. J. 709, 53 (2010).

12. G. P. P. L. Otten, F. Snik, M. A. Kenworthy, M. N. Miskiewicz, and M. J. Escuti, Opt. Express 22, 30287 (2014).

13. E. Vargas Catalán, E. Huby, P. Forsberg, A. Jolivet, P. Baudoz, B. Carlomagno, C. Delacroix, S. Habraken, D. Mawet, J. Surdej, O. Absil, and M. Karlsson, Astron. Astrophys. 595, A127 (2016).

14. A. Aleksanyan and E. Brasselet, Opt. Lett. 41, 5234 (2016).

15. S. Mosca, B. Canuel, E. Karimi, B. Piccirillo, L. Marrucci, R. De Rosa, E. Genin, L. Milano, and E. Santamato, Phys. Rev. A 82, 043806 (2010).

16. E. Khazanov, O. V. Kulagin, S. Yoshida, D. B. Tanner, and D. H. Reitze, IEEE J. Quantum Electron. 35, 1116 (1999).

17. M. Born and E. Wolf, Principles of Optics (Pergamon, 2005).

18. C. Delacroix, O. Absil, P. Forsberg, D. Mawet, V. Christiaens, M. Karlsson, A. Boccaletti, P. Baudoz, M. Kuittinen, I. Vartiainen, J. Surdej, and S. Habraken, Astron. Astrophys. 553, A98 (2013). 